\title{
Synergistic Effect of a Selective Serotonin Reuptake Inhibitor and a Selective Cyclo-0xygenase-2 Inhibitor on Post Spinal Anesthesia Headache
}

\author{
Rivain Fefe Iteke ${ }^{1 *}$, David Mwembo Mutombo ${ }^{2}$, Franck Namegabe Zirhumana ${ }^{1}$, \\ Olivier Kabiriko Mukuku33, Emmanuel Kiyana Muyumba3, Tinsley Mukakala Kibonge ${ }^{2}$, \\ Jean Francois Brichant ${ }^{4}$, Adolphe Manzanza Kilembe5, Yapo Brouh6, \\ Jean Baptiste Zambeze Sakatolo Kakoma ${ }^{3}$
}

\author{
${ }^{1}$ Provincial Hospital of Lubumbashi, Lubumbashi, Congo \\ ${ }^{2}$ Official University of Lubumbashi, Lubumbashi, Congo \\ ${ }^{3}$ University of Lubumbashi, Lubumbashi, Congo \\ ${ }^{4}$ University of Liège, Liège, Belgium \\ ${ }^{5}$ University of Kinshasa, Kinshasa, Congo \\ ${ }^{6}$ Alassane Ouattara University, Abidjan, Cote d'Ivoire \\ Email: *itekefeferivain@gmail.com
}

\begin{abstract}
How to cite this paper: Iteke, R.F., Mutombo, D.M., Zirhumana, F.N., Mukuku, O.K., Muyumba, E.K., Kibonge, T.M., Brichant, J.F., Kilembe, A.M., Brouh, Y. and Kakoma, J.B.Z.S. (2020) Synergistic Effect of a Selective Serotonin Reuptake Inhibitor and a Selective Cyclo-Oxygenase-2 Inhibitor on Post Spinal Anesthesia Headache. Pharmacology \& Pharmacy, 11, 196-208. https://doi.org/10.4236/pp.2020.118018
\end{abstract}

Received: May 5, 2020

Accepted: August 28, 2020

Published: August 31, 2020

Copyright $\odot 2020$ by author(s) and Scientific Research Publishing Inc. This work is licensed under the Creative Commons Attribution International License (CC BY 4.0).

http://creativecommons.org/licenses/by/4.0/

(c) (i) Open Access

\begin{abstract}
Objective: To evaluate the synergistic effect of a Selective Serotonin Reuptake Inhibitor (SSRI) and a Selective Cyclooxygenase 2 Inhibitor (ISCOx-2) in the management of After Rachianesthesia Headache in Obstetric Care. Patients and Methods: This was an experimental double-blind, randomized, multi-center clinical trial of non-inferiority clinical trials over a 12-month period (May 1, 2018 to April 30, 2019) in six hospitals in southeastern DR Congo, including all patients undergoing surgery or analgesia on spinal anesthesia for obstetrical indication and with headache characteristic of a dural breccia, with no known causes, contraindications to experimental products and informed consent to been obtained. The patients were divided into four different groups depending on the protocol used, namely: Group $\mathrm{A}=$ Rehydration + Paracetamol, Group B $=$ Rehydration + Celecoxib, Group C $=$ Rehydration + Nefopam and Group $4=$ Rehydration + Celecoxib + Nefopam (Study Group). Using the Statview II software, the statistical analyzes of data obtained from the different tests were made using the ANOVA test for comparing the averages of different samples using the Fisher test. Verification of the normality of the samples was made by the Kurtosis and Skewness flattening and asymmetry test. Results: The protocol associating Celecoxib and Nefopam signifi-
\end{abstract}


cantly demonstrates its effectiveness in reducing CPRAOs in the first two days with a gradual decrease in anxiety and length of stay. In addition, the majority of patients $(75.6 \%)$ had a high overall level of satisfaction with this protocol. Conclusion: The Nefopam-Celecoxib combination shows its effectiveness in the early reduction of these CPRAOs, anxiety and length of stay with a high overall level of patient satisfaction. Given the good tolerance and accessibility of these products, we strongly suggest the use of this protocol for its validation in our environment and elsewhere, although it is desirable to continue research on other non-explored alien pathways.

\section{Keywords}

Nefopam, Celecoxib, Headache, Rachianesthesia, Obstetrics

\section{Introduction}

Headache after break-in is a complication of spinal anesthesia and an accidental dural breach during anesthesia or epidural analgesia. Their rate of occurrence is higher in the obstetric population with a frequency ranging from $0 \%$ to $46 \%$ [1] [2] [3] [4]. They are responsible for a significant morbidity and prolongation of hospitalization with a considerable socio-economic, psychological and professional impact [1] [3] [5]. The leakage of the Cerebro-Spinal Fluid (LCS) caused by the dural breach is responsible for a decrease in the volume and pressure of the LCS, which explains the traction on intracranial nerve structures at orthostatism [4] [6] [7]. This breach is often accompanied by small nerve damage involving several peripheral and central pain signaling pathways [2] [4]. Several preventive and curative measures have been studied, but none has proven to be effective enough except the Epidural Blood patch, which was not the only treatment of choice given that rehydration and other drugs like sumatriptan, morphine drugs, adrenocorticotropic hormone (ACTH), opiates, nonsteroidal anti-inflammatory drugs have been tested in isolation leading to controversial results [1] [2] [8] [9]. This therapeutic difficulty remains a serious problem, especially for countries with limited resources where these headaches are increasing rapidly in obstetric settings (27.5\%), although this same study in DR Congo has just developed a reproducible predictive score from determinants specific to the environment and the obstetrical population [10]. If this score could make it possible to detect these cases early, a preventive treatment a little more effective than the existing measures, would be possible. It is true that currently few studies have been pursued, particularly in the search for the synergistic effect of existing measures, admitted to being ineffective in isolation, in order to reduce the use of the blood patch to some extent or even the heavy psycho-socio-economic burden that they train. Knowing today the remarkable benefit of a combination of analgesics acting at the same time on several pain signaling pathways and thus subscribing to the concept of "multimodal or balanced" analgesia, it seems en- 
couraging to us to test any possible analgesic synergy on these headaches in this obstetric population which remains the most affected. This is why in order to contribute to the resolution of this worrying problem in our environment and mainly in obstetrics, we proposed to conduct this study whose objective is to assess the synergistic effect of a Selective Inhibitor Serotonergic Recapture (SSRI) and a Selective Cyclo-Oxygenase 2 Inhibitor (ISCOx-2) in the management of CPRAO.

\section{Materials and Methods}

\section{Type and period of study}

This was a 12-month, double-blind, non-inferiority, multi-center randomized controlled clinical trial type study (May 1, 2018 to April 30, 2019).

Study sites

This work was carried out in 6 hospitals in south-eastern DR Congo (Hôpital Général Charité Maternelle and Hôpital Kyeshero de Goma, Hôpital de Minova and Center Hospitalier Skyborne du Sud-Kivu, as well as the Sendwe Provincial Reference Hospital and Medical Center Diamond of Lubumbashi). The choice of these structures was motivated by their large reception capacity, their academic vocation, as well as by an increased and coordinated obstetric anesthesia practice.

\section{Inclusion criteria}

All patients operated on or analgesized under spinal anesthesia for an obstetric indication and having presented the headaches characteristic of a dural brachial breach, with no other known causes, presenting contraindications to the experimental products and whose informed consent has been obtained were included in this study.

Exclusion criteria

All patients were excluded from the study:

- Having undergone spinal anesthesia for a non-obstetric indication;

- Having presented headaches that are not characteristic or related to another known cause;

- Having absolute contraindications to the products used for the clinical study;

- Not having given their consent.

Products used and methods of administration

Nefopam: UIPAC name (RS) - 5-methyl-1-phenyl-1,3,4,6-tetrahydro-2,5 Benzoxazocine whose chemical formula is C17H19NO. It is a non-opioid analgesic, used for the treatment of pain of moderate to severe intensity, marketed under the name of Acupan ${ }^{\infty}$. It is part of level 1 of the classification of analgesics according to the WHO. Its analgesic effect consists in a selective inhibition of the reuptake of serotonin and catecholamines. Administration is by intramuscular and slow intravenous route practically at a dosage of $20 \mathrm{mg}$ every 6 hours without exceeding $120 \mathrm{mg}$ per 24 hours and its mean plasma half-life is 5 hours. It is contraindicated in cases of: hypersensitivity to Nefopam or one of its constitu- 
ents before 15 years of age or in the event of convulsions or a history of convulsive disorders.

Certain complications are reported in particular: urinary retention and the risk of glaucoma by closing the angle.

In addition, it is necessary to pay attention to the additive effects of other molecules, in particular on their depressant effects of the central nervous system which can thus contribute to decrease vigilance. Impaired alertness can make driving and using machines dangerous [11].

Celecoxib

Celecoxib is an oral, selective inhibitor of cyclooxygenase-2 (COX-2) which is responsible for the synthesis of prostaglandins. Two isoforms, COX-1 and COX-2, have been identified. Cyclo-oxygenase-2 (COX-2) is the isoform of the enzyme induced by pro-inflammatory stimuli and is believed to be the main responsible for the synthesis of prostanoid mediators of pain and inflammation.

At subclinical doses, Celecoxib had no effect on platelet aggregation, alteration of the gastric mucosal barrier, or on bleeding time compared to placebo [12].

\section{Study groups}

Based on different evaluations to be carried out, we had formed 4 study groups distributed below according to their respective protocols as well as the evaluation parameters from the onset of headache until the discharge of patients (Figure 1).

\section{Conduct of the study and data collection}

Work had started during the same period at different sites. The collection of cases was continuous and all cases of the CPRAO were notified to the focal point

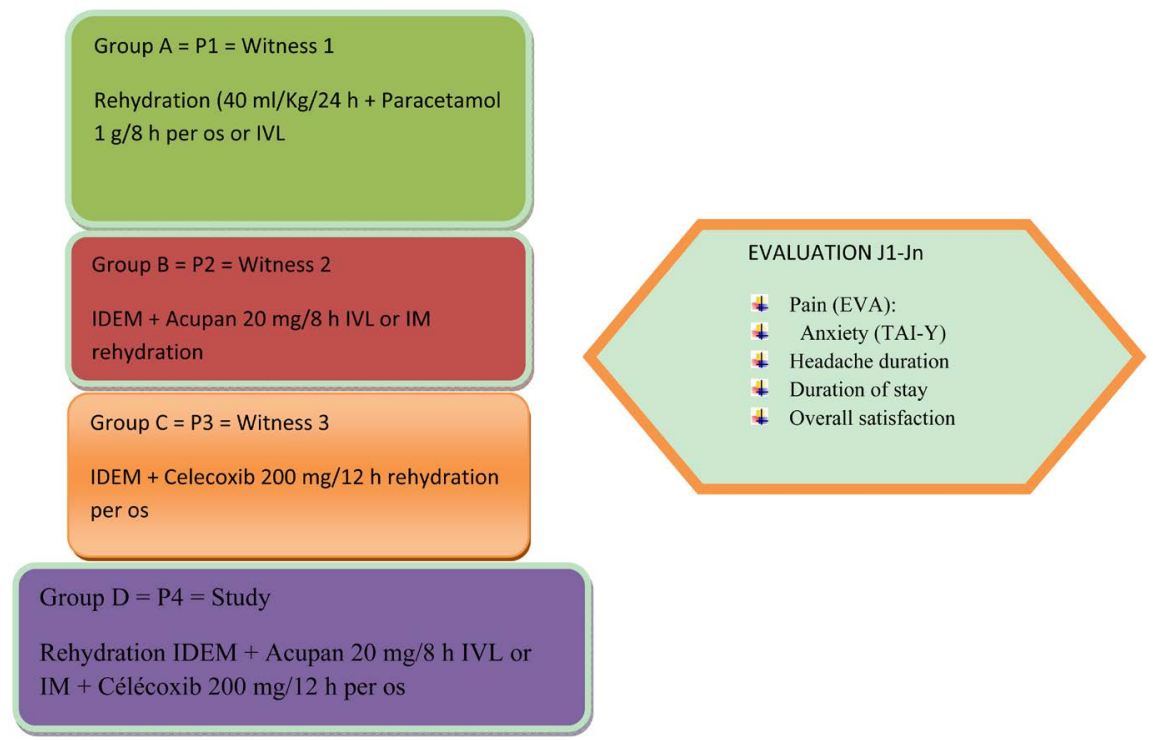

Figure 1. Distribution partition of groups according to different protocols $\mathrm{P}=$ Therapeutic Protocol; EVA = Analog Visual Scale; TSAI-Y = Anxiety Scale; IVL = Slow Intravenous; IM = Intramuscular. 
of the site who confirmed the case by a complete clinical examination and decided whether to include or not the case. The products of the experimental protocol were free throughout the study period. Then, he indicated a protocol by leaving the EVA strip and the TSAI-Y questionnaire at the patient's bedside. The protocol to be followed was recorded in the patient's file and followed by the site nurses and investigative doctor who were automatically part of the obstetrics or anesthesia department. The evaluation was done every morning around the room and the observations were reported in the file as well as the data collection register kept by the site investigator. All cases of headache persistence requiring further action were discussed either directly, on the phone or by email with the principal investigator, as we toured the sites weekly although we had weekly feedback. Finally, before the patient leaves, the site investigator will complete the length of stay, headache and overall patient satisfaction.

All of this data was centralized and encoded in an Excel file for further processing.

Assessment of efficacy and tolerance

Pain Intensity (Headache): VAS was used because it is among the simple and reproducible reference tools in the assessment of pain in adults. It is in the form of a continuous scale not graduated on the side presented to the patient and graduated from 0 to $100 \mathrm{~mm}$ on the side of the caregiver. Using a cursor, the patient designated the intensity of the pain felt, the back of the strip allowing the clinician to schematically quantify this data.

Dimensions of anxiety: This was a self-assessment questionnaire (individually or in groups) of the dimensions of anxiety of any origin. STAY forms Y-A (state anxiety) with 20 propositions to know what the subject is feeling at the time, then STAY forms Y-B (trait anxiety) also with 20 propositions to know what the subject generally feels. Each item has a score ranging from 1 to 4 (4 being the highest level of anxiety). It is rated as follows: Very high: $>65$; High: from 56 to 65; Medium: from 46 to 55; Low: from 36 to 45 and Very low: $<$ or $=35$.

\section{Primary endpoint}

The main endpoint representing the variable to be explained was the effectiveness of the combination of Nefopam and Celecoxib in the management of CPRAO.

\section{Secondary endpoints}

Secondarily, other criteria were judged, in particular: the reduction in anxiety associated with these headaches, the duration of the headaches under treatment, the length of stay as well as the level of overall satisfaction of the patients.

\section{Criteria for leaving the study}

All patients who presented with a serious complication, those who stopped or refused treatment at the start of the study, or those who refused to be evaluated during the study were considered not to be part of the study.

Patient safety

All pharmacovigilance recommendations for these different products were 
well explained to the investigators so that any attributable adverse reaction was managed according to the protocol in the pharmacovigilance guide, both in the leaflets and in the available compendium.

Statistical analyzes

Using Statview II software, statistical analyzes of data obtained from the different tests were done using the ANOVA test to compare the means of different samples using the Fisher test to better explain the statistically significant difference between the distributions. The normality of the samples was checked by the Kurtosis and Skewness flattening and asymmetry test. In order to determine if, after a significant ANOVA, the means of the groups of the independent variable differ, the HSD Tukey and Bonferroni-Sheffé post hoc test were used to better control the experimental error rate at a well defined threshold, but also not requiring an identical sample size in the different groups. The significance level was set at $\mathrm{p}<0.0001$ corresponding to a very highly significant difference. All the results were expressed in the form of Averages \pm ESM (Standard Error on the Average).

Ethical and regulatory aspects

This work had received the approval of the ethics committee of the University of Lubumbashi in DR Congo ( $\mathrm{n}^{\circ}$ Approval: UNILU/CEM/170/2019) and since its preparation until its submission various provisions in terms of compliance with human life were scrupulously respected, in particular those linked to:

- The Helsinki Declaration and Good Clinical Practice;

- Patient information and obtaining informed consent;

- The favorable opinion of the personal protection committees.

\section{Results}

\section{1) Workforce}

Our overall number was 165 patients randomly divided into 4 different groups $(\mathrm{A}=37, \mathrm{~B}=42, \mathrm{C}=45, \mathrm{D}=41)$.

2) Evaluation of the intensity of headache postoperatively according to the analgesic protocols used (Tables 1-5)

3) Evaluation of the length of post-operative stay of the operated patients having presented CPRAO according to the protocols used (Table 6)

4) Assessment of the level of anxiety of the operated patients having presented CPRAO according to the protocols used (Tables 7-9)

Line in the middle of the rectangle: median; Line at the bottom of the rectangle: first quartile (25\%); Line in the upper part furthest from the rectangle: third quartile (75\%); Item: outlier; Lowest line: lowest value (minimum), excluding outliers; Line at the extreme top: highest value (maximum), excluding outliers. (Figure 2).

The combination Nefopam-Celecoxib (Pr4) had significantly reduced the level of anxiety from the first day with a significant decline by the third, and almost zero by the fourth-fifth day. 


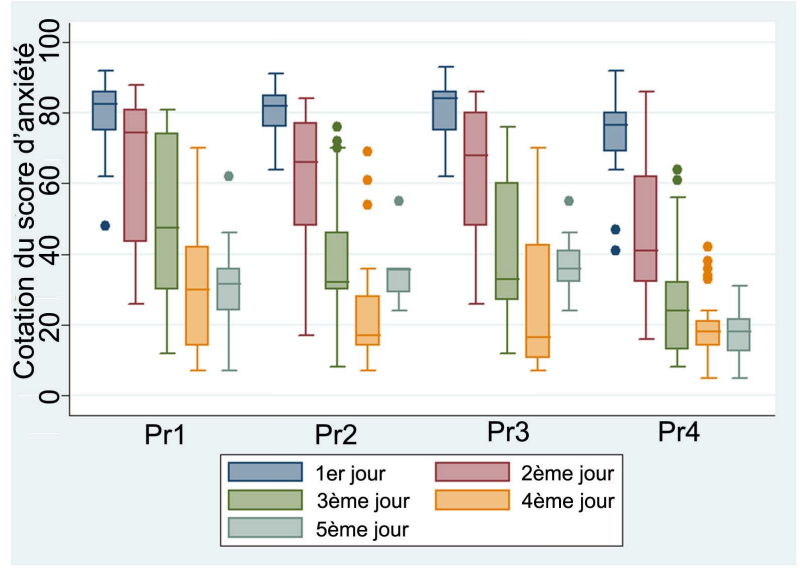

Figure 2. Rating of the anxiety score in cesareans according to the anesthetic protocol used.

Table 1. Evaluation of the simple visual scale in patients operated on the first day according to the anesthetic protocol used.

\begin{tabular}{ccrrrrrrr}
\hline Protocol & \multicolumn{2}{c}{ Low } & \multicolumn{2}{c}{ Moderate } & \multicolumn{2}{c}{ Intense } & Total & p \\
\hline Pr1 & 2 & $5.41 \%$ & 3 & $8.11 \%$ & 32 & $86.49 \%$ & 37 & \\
Pr2 & 1 & $2.38 \%$ & 9 & $21.43 \%$ & 32 & $76.19 \%$ & 42 & \\
Pr3 & 0 & $0.00 \%$ & 7 & $15.56 \%$ & 38 & $84.44 \%$ & 45 & $<0.0001$ \\
Pr4 & 6 & $14.63 \%$ & 22 & $53.66 \%$ & 13 & $31.71 \%$ & 41 & \\
\hline
\end{tabular}

Compared to other protocols, the Nefopam-Celecoxib ( $\operatorname{Pr} 4)$ combination significantly reduced headache from the first day because it was less intense in patients (31.7\%), more moderate (53.7\%) and weaker (14.6\%).

Table 2. Evaluation of the simple visual scale in patients operated on the 2nd day according to the anesthetic protocol used.

\begin{tabular}{ccccccccc}
\hline Protocol & \multicolumn{2}{c}{ Low } & \multicolumn{2}{c}{ Moderate } & \multicolumn{2}{c}{ Intense } & Total & $\mathrm{p}$ \\
\hline $\operatorname{Pr} 1$ & 1 & 13 & $35.14 \%$ & $2.70 \%$ & 23 & $62.16 \%$ & 37 & \\
$\operatorname{Pr} 2$ & 4 & 19 & $45.24 \%$ & $9.52 \%$ & 19 & $45.24 \%$ & 42 & \\
$\operatorname{Pr} 3$ & 3 & 15 & $33.33 \%$ & $6.67 \%$ & 27 & $60.00 \%$ & 45 & $<0.0001$ \\
$\operatorname{Pr} 4$ & 26 & 13 & $31.71 \%$ & $63.41 \%$ & 2 & $4.88 \%$ & 41 & \\
\hline
\end{tabular}

On the second day, there were significantly fewer patients with intense headache (4.9\%) and more mild headache with Pr4.

Table 3. Evaluation of the simple visual scale in 3rd day open as according to the anesthetic protocol used.

\begin{tabular}{ccccccccc}
\hline Protocole & \multicolumn{2}{c}{ Low } & \multicolumn{2}{c}{ Moderate } & \multicolumn{2}{c}{ Intense } & Total & $\mathrm{p}$ \\
\hline $\operatorname{Pr} 1$ & 3 & $8.11 \%$ & 26 & $70.27 \%$ & 8 & $21.62 \%$ & 37 & \\
$\operatorname{Pr} 2$ & 22 & $52.38 \%$ & 17 & $40.48 \%$ & 3 & $7.14 \%$ & 42 & \\
$\operatorname{Pr} 3$ & 11 & $24.44 \%$ & 30 & $66.67 \%$ & 4 & $8.89 \%$ & 45 & $<0.0001$ \\
$\operatorname{Pr} 4$ & 35 & $85.37 \%$ & 6 & $14.63 \%$ & 0 & $0.00 \%$ & 41 & \\
\hline
\end{tabular}

It appears on the third day that with $\operatorname{Pr} 4$ compared to the other three protocols, no patient with severe headache, only (14.6\%) with moderate headache and almost all (85.4\%) of patients with low intensity headache. 
Table 4. Evaluation of the simple visual scale in patients operated on the 4 th day according to the anesthetic protocol used.

\begin{tabular}{|c|c|c|c|c|c|c|c|c|}
\hline Protocol & \multicolumn{2}{|c|}{ Nothing } & \multicolumn{2}{|c|}{ Low } & \multicolumn{2}{|c|}{ Moderate } & \multirow{2}{*}{$\begin{array}{c}\text { Total } \\
37\end{array}$} & $\mathrm{p}$ \\
\hline Pr1 & 0 & $0.00 \%$ & 21 & $56.76 \%$ & 16 & $43.24 \%$ & & \multirow{4}{*}{$<0.0001$} \\
\hline Pr2 & 4 & $9.52 \%$ & 30 & $71.43 \%$ & 8 & $19.05 \%$ & 42 & \\
\hline Pr3 & 3 & $6.67 \%$ & 26 & $57.78 \%$ & 16 & $35.56 \%$ & 45 & \\
\hline Pr4 & 10 & $24.39 \%$ & 31 & $75.61 \%$ & 0 & $0.00 \%$ & 41 & \\
\hline
\end{tabular}

A quarter of the patients no longer had headaches by the fourth day and the rest had only mild headaches with Pr4 compared to other protocols.

Table 5. Evaluation of the simple visual scale in patients operated on the 5th day according to the anesthetic protocol used.

\begin{tabular}{ccccccccc}
\hline Protocol & \multicolumn{2}{c}{ Nothing } & \multicolumn{2}{c}{ Low } & \multicolumn{2}{c}{ Moderate } & \multicolumn{2}{c}{ Total } \\
\hline Pr1 & 19 & $51.35 \%$ & 17 & $45.95 \%$ & 1 & $2.70 \%$ & 37 & \\
Pr2 & 29 & $69.05 \%$ & 11 & $26.19 \%$ & 2 & $4.76 \%$ & 42 & $<0.0001$ \\
Pr3 & 23 & $51.11 \%$ & 19 & $42.22 \%$ & 3 & $6.67 \%$ & 45 & 41
\end{tabular}

On the fifth day, a complete absence of headache was significantly noted in the majority of patients $(70.3 \%)$ and only a quarter of patients with mild headache with Pr4.

Table 6. Hospital stay (in days) of operated patients according to the anesthetic protocol used.

\begin{tabular}{ccccccc}
\hline Protocol & Effective & Average & Standard deviation & Quartile 1 & Médiane & Quartile 3 \\
\hline Pr1 & 36 & 7.7 & 1.8 & 7 & 7 & 8 \\
Pr2 & 40 & 7.1 & 1.2 & 6 & 7 & 8 \\
Pr3 & 35 & 7.3 & 1.6 & 6 & 7 & 8 \\
Pr4 & 40 & 6.3 & 1.1 & 5.5 & 6 & 7 \\
\hline
\end{tabular}

ANOVA test: $\mathrm{F}=6.02 ; \mathrm{p}<0.0001$. The mean length of stay was low (6.3 days) with Pr4 compared to other protocols

Table 7. Rating of the anxiety score in patients operated on according to the anesthetic protocol used on the first day.

\begin{tabular}{ccccccc}
\hline Protocol & Effective & Average & Standard deviation & Quartile 1 & Médiane & Quartile 3 \\
\hline Pr1 & 36 & 79.4 & 9.5 & 75 & 82.5 & 86 \\
Pr2 & 42 & 80.1 & 7.2 & 76 & 82 & 85 \\
Pr3 & 42 & 81.5 & 7.8 & 75 & 84 & 86 \\
Pr4 & 41 & 74.7 & 10.1 & 69 & 76.5 & 80 \\
\hline
\end{tabular}

ANOVA test: $\mathrm{F}=4.61 ; \mathrm{p}=0.004$. The anxiety score showed a moderate level (74.1) with $\operatorname{Pr} 4$ compared to other protocols from the first day of treatment.

5) Evaluation of the overall level of satisfaction of the operated patients who presented CPRAO according to the protocols used (Table 10) 
Table 8. Rating of the anxiety score in cesareans according to the anesthetic protocol used on the 2 nd day.

\begin{tabular}{ccccccc}
\hline Protocol & Effective & Average & Standard deviation & Quartile 1 & Médiane & Quartile 3 \\
\hline Pr1 & 36 & 65.1 & 18.8 & 43.5 & 74.5 & 81.0 \\
$\operatorname{Pr} 2$ & 42 & 62.3 & 16.9 & 48.0 & 66.0 & 77.0 \\
$\operatorname{Pr} 3$ & 42 & 64.3 & 17.2 & 48.0 & 68.0 & 80.0 \\
$\operatorname{Pr} 4$ & 41 & 45.3 & 18.5 & 32.0 & 41.0 & 62.0 \\
\hline
\end{tabular}

ANOVA test: $\mathrm{F}=11.17 ; \mathrm{p}<0.0001$. A low level of anxiety was significantly observed already on the second day in patients on $\operatorname{Pr} 4$.

Table 9. Rating of the anxiety score in cesareans according to the anesthetic protocol used on the 3rd day.

\begin{tabular}{ccccccc}
\hline Protocole & Effective & Average & Standard deviation & Quartile 1 & Médiane & Quartile 3 \\
\hline Pr1 & 36 & 48.4 & 22.5 & 30.0 & 47.5 & 74.0 \\
Pr2 & 42 & 37.6 & 18.8 & 30.0 & 32.0 & 46.0 \\
Pr3 & 42 & 41.6 & 21.2 & 30.0 & 33.0 & 60.0 \\
Pr4 & 41 & 26.9 & 16.3 & 13.0 & 24.0 & 32.0 \\
\hline
\end{tabular}

ANOVA test: $\mathrm{F}=8.05 ; \mathrm{p}<0.0001$.

Table 10. Overall level of satisfaction of caesareans according to the anesthetic protocol used.

\begin{tabular}{cccccccc}
\hline \multirow{2}{*}{ Protocol } & \multicolumn{5}{c}{ Overall level of satisfaction } & \multirow{2}{*}{ Total } \\
\cline { 2 - 6 } & \multicolumn{2}{c}{ Low } & \multicolumn{2}{c}{ Medium } & \multicolumn{2}{c}{ Large } & \\
\hline Pr1 & 14 & $(37.84 \%)$ & 22 & $(59.46 \%)$ & 1 & $(2.70 \%)$ & 37 \\
Pr2 & 7 & $(17.95 \%)$ & 27 & $(64.29 \%)$ & 8 & $(19.05 \%)$ & 42 \\
Pr3 & 10 & $(27.03 \%)$ & 27 & $(61.36 \%)$ & 7 & $(15.91 \%)$ & 44 \\
$\operatorname{Pr} 4$ & 0 & $(0.00 \%)$ & 10 & $(24.39 \%)$ & 31 & $(75.61 \%)$ & 41
\end{tabular}

Patients on Pr4 showed a high overall level of satisfaction compared to those on other protocols. Fisher test: $\mathrm{p}<0.0001$

\section{Discussion}

The pathophysiology of post-spinal anesthesia headache remains complex today, especially with regard to the various pain signaling pathways, making analgesic treatment random. Nevertheless, it is known that the meningeal breach is responsible for a reduction in the volume of Cerebrospinal Fluid which, among other things, acts as a hydraulic shock absorber for the brain in the cranial box. During the transition to orthostatism, the craniocaudal displacement of the encephalon is no longer absorbed by the liquid column. The brain comes crashing down on the base of the skull, whose various asperities (particularly the edge of the rock and the cavernous sinus) will be responsible for localized suffering following an inflammatory cascade as well as the potentiation of the efferent pathways of pain. This displacement of the brain puts in tension the vasculofibrous 
meningeal structures of the convexity, tension which is partly responsible for the headache, by the activation of the stretch-sensitive receptors meningeal at the level of the trigeminal nerves (frontal headaches), glossopharyngeal and Wave (occipital headache), and the first three cervical roots [9].

In fact, from this energization, the neurons with convergence of the posterior horn are very strongly inhibited when a nociceptive stimulation is applied to any part of the body, different from their excitatory peripheral field: this stimulation triggers CIDN. The CIDN are underpinned by a complex loop involving supra-spinal structures belonging to the bulbar reticulate formation such as the subnucleus reticularis dorsalis (SRD). Neuromediators are endorphinic and serotonergic [13]. Several therapies have been tried, but have been shown to be ineffective in the cause, or even ineffective in symptomatology. Among these we can cite:

- Paracetamol which was the basic analgesic treatment, effective as long as its administration is continued. It just minimizes the headache without healing it.

- Codeine, an adjuvant to paracetamol, is not recommended because its side effects (nausea, vomiting) increase the patient's discomfort and complicate the diagnosis. In addition, all morphine derivatives are vasodilators, which can increase headache. Other opiates such as tramadol have no justification in this context, and their possible side effects (nausea and vomiting) contraindicate them [14] [15] [16].

- Caffeine remains a subject of controversy [17]. Its action is explained by a vasoconstrictor effect on the cerebral circulation. If this treatment is based on a suitable mechanism, it is only moderately effective clinically. Within 24 hours of stopping treatment, the headache recurs in more than $50 \%$ of patients. The only controlled study reports no benefit from caffeine, regardless of the dosage. In addition, side effects, lack of sleep, tachycardia and hypertension, are frequent [14].

- Nonsteroidal anti-inflammatory drugs (NSAIDs) are effective as analgesics, without any efficacy on the cause. The NSAIDs chosen will be of the anti-Cox2 type, with a short duration of action, not prohibiting the production of a blood-patch. Non-reversible long-acting NSAIDs and aspirin are disqualified. They do not bring any improvement and interfere with the realization of a blood-patch [14] [15] [18].

This orientation was the basis for the choice in our clinical trial of a short-acting selective COX-2 inhibitor NSAID, Celecoxib, which was initially evaluated alone with a placebo in group B, but had not shown its superiority compared to the neutral group, the Nefopam group alone as well as the Nefopam-Celecoxib combination. On the other hand, its association with Nefopam had shown effectiveness in terms of considerable reduction in pain, anxiety and the length of post-operative stay. This could be explained by the fact that COX-2, originally detected in monocytic cells activated by cytokines, is above all induced by certain stimuli which increase its tissue expression, particularly during the inflam- 
matory response where its activity increases more than twenty times, which would show a certain effectiveness on the selective inhibition of endoperoxides at the peripheral level, thus preventing the activation of the afferent pathways. This action would be complemented synergistically by the effect of Nefopam which selectively inhibits serotonergic reuptake, thus exerting inhibitory control on non-specific medullary nociceptive neurons [16] [19]. Non-selective serotonergic reuptake inhibitors such as triptans have not been shown to be effective and are not used clinically [19] [20] [21].

The problem noted in the results of all of this previous work was not to associate certain analgesics acting on the main pathways strongly involved in the occurrence or maintenance of these headaches. Although much remains to be done on the perfect mastery of all the pain signaling pathways in this pathophysiology, our study had proposed to associate a selective serotonergic reuptake inhibitor involved in the inhibition of the descending pathways of pain and a much more selective cyclooxygenase inhibitor involves local and central inhibition of inflammatory pain.

Very recently, other effects of Nefopam have been demonstrated, in particular in the inhibition of the release of glutamate, a neurotransmitter activating the $\mathrm{N}$-methyl-d-Aspartate receptors (NMDA) post-synaptic reducing neuronal excitability and hyperalgesia towards the supramedullary structures. This complexity of action on the pain of these two molecules is further reinforced by their selectivity, covering in addition to the descending and inflammatory monoaminergic pathways, other pathways such as postsynaptic NMDA and glutamate whose role in the pain modulation is well known today [20]. This work would open other doors of research, especially with regard to certain combinations of analgesics depending on possible incriminating pathways in the pathophysiology of these headaches, because it emerges: a reduction in pain already from the first day onset of pain until no patient with severe pain by the third day of treatment and less than $15 \%$ with moderate pain. This protocol also significantly reduced anxiety until a slight note by the second and third day. This had reduced the stay from one to two days compared to other protocols with an overall satisfaction level of more than $75 \%$. In view of the various pathophysiological considerations, the effectiveness of this protocol is justified by this, probably covering several pain signaling pathways compared to selective or non-selective monotherapy. This seems very encouraging not only by the effective progressive reduction of pain, but also and above all the anxiety which accompanies it, as well as the length of stay somewhat resolving the socio-economic cost of these headaches. Furthermore, no major side effects have been reported in this combination and these products are readily available in our environment.

\section{Conclusion}

The CPRAO is still a major problem in our environment, although the determinants are known in the obstetric environment with a predictive score available. With regard to existing drug therapies, the Nefopam-Celecoxib association shows 
its effectiveness in the early reduction of these CPRAOs, anxiety as well as length of stay with a high overall level of patient satisfaction. Given the good tolerance and accessibility of these products, we strongly suggest the use of this protocol for its validation in our environment and elsewhere, although it is desirable to continue research on other non-exploding allogenic pathways.

\section{Acknowledgements}

The authors thank all hospitals in DR Congo, as well as the University of Lubumbashi for accepting that this work is part of our aggregation thesis. To Dr Samantha Sparow, we say thank you for the English translation of the manuscript.

\section{Conflicts of Interest}

The authors do not declare any conflict of interest throughout the course of this work.

\section{References}

[1] Lenelle, L., Lahaye-Goffart, B., Dewandre, P.Y. and Brichant, J.F. (2011) Les céphalées post-ponction durale: Traitement et prévention. Revue Médicale de Liège, 66, 575-580.

[2] Marie-Pierre, B., et al. (2016) Que faire en cas de brèche de la dure-mère en salle de travail? Le praticien en anesthésie réanimation, 20, 61-65. https://doi.org/10.1016/j.pratan.2016.02.009

[3] Zué, A.S., Ntamack, J.B. and Biyogo, P. (2009) Management of Headache after Spinal Anesthesia in an African Hospital. Journal Canadien D' anesthesie, 56, 549-550. https://doi.org/10.1007/s12630-009-9100-3

[4] Sachs, A. and Smiley, R. (2014) Post-Dural Puncture Headache: The Worst Common Complication in Obstetric Anesthesia. Seminars in Perinatology, 38, 386-394. https://doi.org/10.1053/j.semperi.2014.07.007

[5] Heesen, M., Kloehr, S., Rossaint, R., Van De Velde, M. and Straube, S. (2013) Can the Incidence of Accidental Dural Puncture in Laboring Women Be Reduced? A Systematic Review and Meta-Analysis. Minerva Anestesiologica, 79, 1187-1197.

[6] Curelaru, I. (1979) Long Duration Subarachnoid Anesthesia with Continuous Epidural Blocks. Praktische Anästhesie, Wiederbelebung und Intensivtherapie, 14, 71-78.

[7] Fournet-Fayard, A. and Malinovsky, J.M. (2013) Céphalées post-brèches méningées et blood-patch: Aspects théoriques et pratiques. Annales Françaises d'Anesthésie et de Réanimation, 32, 325-338. https://doi.org/10.1016/j.annfar.2013.02.014

[8] Coates, M.B. (1982) Combined Subarachnoid and Epidural Techniques. Anaesthesia, 37, 89-90. https://doi.org/10.1111/j.1365-2044.1982.tb01016.x

[9] Zetlaoui, P.J. (2010) Céphalées par hypotension du liquide cérébrospinal. In: Brasseur, L. and Djianéds, M.-C., Eds., Douleurs. Techniques Invasives, Arnette, Paris, 157-184.

[10] Iteke, F.R., Brouh, Y., Brichant, J.F., Kakoma, S.Z., et al. (2019) Post-Spinal Anesthesia Headaches in Obstetric Resource-Limited Settings: Epidemiological and Clinical Characteristics, Determinants and Predictive Score. Anesthesia \& Pain Research, 3, 1-6.

[11] Chan, F., Lanas, A., Scheiman, J., Berger, M.F., Nguyen, H. and Goldstein, J.L. 
(2010) Celecoxib versus Omeprazole and Diclofenac in Patients with Osteoarthritis and Rheumatoid Arthritis (CONDOR): A Randomised Trial. The Lancet, 376, 173-179. https://doi.org/10.1016/S0140-6736(10)60673-3

[12] Sanga, M., Banach, J., Ledvina, A., Modi, N.B. and Mittur, A. (2016) Pharmacokinetics, Metabolism, and Excretion of Nefopam, a Dual Reuptake Inhibitor in Healthy Male Volunteers. Xenobiotica, 46, 1001-1016.

[13] Melzack, R., Weisz, A.Z. and Sprague, L.T. (1963) Stratagems for Controlling Pain: Contributions of Auditory Stimulation and Suggestion. Experimental Neurology, 8, 239-247. https://doi.org/10.1016/0014-4886(63)90034-7

[14] Boonmak, P. and Boonmak, S. (2010) Epidural Blood Patching for Preventing and Treating Post-Dural Puncture Headache. Cochrane Database of Systematic Reviews, 2, CD001791. https://doi.org/10.1002/14651858.CD001791.pub2

[15] Al-Metwalli, R.R. (2008) Epidural Morphine Injections for Prevention of Postdural Puncture Headache. Anaesthesia, 63, 847-850. https://doi.org/10.1111/j.1365-2044.2008.05494.x

[16] Apfel, C.C., Saxena, A., Cakmakkaya, O.S., et al. (2010) Prevention of Postdural Puncture Headache after Accidental Dural Puncture: A Quantitative Systematic Review. British Journal of Anaesthesia, 105, 255-263.

https://doi.org/10.1093/bja/aeq191

[17] Naja, Z., Al-Tannir, M., El-Rajab, M., Ziade, F. and Baraka, A. (2009) Nerve Stimulator-Guided Occipital Nerve Blockade for Postdural Puncture Headache. Pain Practice, 9, 51-58. https://doi.org/10.1111/j.1533-2500.2008.00238.x

[18] Connelly, N.R., Parker, R.K., Rahimi, A. and Gibson, C.S. (2000) Sumatriptan in Patients with Postdural Puncture Headache. Headache, 40, 316-319. https://doi.org/10.1046/j.1526-4610.2000.00047.x

[19] Basurto Ona, X., Martínez García, L., Solà, I. and Bonfill Cosp, X. (2011) Drug Therapy for Treating Postdural Puncture Headache. Cochrane Database of Systematic Reviews, No. 8, CD007887. https://doi.org/10.1002/14651858.CD007887.pub2

[20] Verleye, M. and Gillardin, J.M. (2009) Contribution of Transient Receptor Potential Vanilloid Subtype 1 to the Analgesic and Antihyperalgesic Activity of Nefopam in Rodents. Pharmacology, 83, 116-121. https://doi.org/10.1159/000186050

[21] Gray, A., Nevinson, M. and Sewell, R. (1999) The Involvement of Opiodergic and Noradrenergic Mechanisms in Nefopam Antinociception. European Journal of Pharmacology, 365, 149-157. https://doi.org/10.1016/S0014-2999(98)00837-1 